\title{
Ю.Д. Чашечкин
}

\section{ЛИГАМЕНТЫ И ВОЛНЫ В ПЕРИОДИЧЕСКИХ ТЕЧЕНИЯХ}

\author{
Институт проблем механики им. А.Ю. Иилинского РАН \\ Россия, 119526, Москва, проспект Вернадского, 101/1, \\ Тел.: 8(495)434-00-17, E-mail: ipmnet@ipmnet.ru
}

\begin{abstract}
Представлены результаты теоретических и лабораторных исследований динамики и структуры периодических течений - капиллярных и акустических волн импакта капли на основе системы фундаментальных уравнений механики жидкостей. Система включает уравнения состояния для потенциала Гиббса и его производных - термодинамических параметров, а также уравнения переноса вещества, импульса, энергии. Решение строится методами теории сингулярных возмущений с учетом условия совместности. Приведены полные решения задач распространения линейных акустических, внутренних и гибридных волн. Впервые рассмотрены и регулярные (волновые), и сингулярные решения, характеризующие лигаменты - тонкие волокна и прослойки. Прослежены процессы формирования структур двумерных и трехмерных периодических и присоединенных внутренних волн. В течениях, порождаемых в жидкости свободно падающими каплями, прослежена связь капиллярных волн с процессами излучения звуковых пакетов.
\end{abstract}

Ключевые слова: лигаменты, волны, динамика и структура течений, внутренние волны

\section{Введение}

Исследования волн - периодических течений, в которых параметры мгновенной пространственной структуры переменных физических полей (волновое число $\mathbf{k}$ ) связаны с локальными характеристиками временной изменчивости (частотой $\omega$ ) функциональным (дисперсионным) соотношением $\omega=\omega \mathbf{k}$, активно проводятся во многих областях науки и техники. Распространенные в природе виды волн - гравитационные (поверхностные и внутренние), акустические, капиллярные, инерциальные, гибридные и другие - обычно описываются специализированными моделями («волновыми уравнениями»), полученными в приближениях идеальной или вязкой жидкостей [1]. В последние годы на основе анализа полной системы фундаментальных уравнений механики жид- 
костей методами теории сингулярных возмущений в линейном и слабо нелинейном приближениях развивается новая теория периодических течений [2].

Классификация компонентов периодических течений. Ранг полной нелинейной системы, порядок ее линеаризованной версии и степень алгебраического (дисперсионного) соотношения, задаваемые условиями взаимности, зависят от полноты учета физических свойств среды - эффектов стратификации, сжимаемости и диссипации (вязкости, диффузии и температуропроводности), определяют число (минимальное для нелинейных систем) независимых функций, составляющих полное решение. При этом регулярные решения характеризуют волны, а семейства сингулярно возмущенных решений - лигаменты - математические образы тонких прослоек и отдельных волокон, формирующих собственную тонкую структуры среды. Оценки поперечных масштабов компонентов, периодических течений различных типов - гравитационных, акустических, инерциальных и гибридных - приведены в [3]. Их длина определяется условиями задачи и диссипативными свойствами сред.

Проведенный анализ показал, что системы фундаментальных уравнений, учитывающие эффекты стратификации (расслоения сред, неоднородных по температуре и/или по концентрации в поле массовых сил) и диссипации корректно поставлены и разрешимы [2]. В приближении постоянной плотности жидкости разрешимы только двумерные модели, трехмерные задачи переопределены и для сжимаемых, и для несжимаемых сред.

Все течения, динамика которых описывается полным набором решений системы фундаментальных уравнений, включающих функции с несоизмеримыми пространственными и временными масштабами, структурированы и нестационарны. Традиционные волновые уравнения - редуцированные версии фундаментальной системы - не позволяют рассчитывать тонкую структуру возмущений исследуемых полей, формулировать обоснованные метрологические требования к методикам лабораторных и натурных измерений.

Системы фундаментальных уравнений механики жидкостей позволяют решать задачи генерации, распространения, отражения от твердых стенок и свободной поверхности и распада различных типов волн с физически обоснованными начальными и граничными условиями. Расчет задачи генерации периодических внутренних волн вертикально осциллирующим диском выполнен в [4].

Численный анализ аналитического решения показал, что как источник, так и пучок бегущих внутренних волн, оконтурены лигамен- 
тами - семействами тонких оболочек, степень компактности и геометрия которых синхронно меняются с фазой волны. Поперечный масштаб лигаментов, который определяется частотой волны $\omega$ и величиной диссипативных коэффициентов, кинематической вязкости $v$, температуропроводности $\kappa_{T}$ и диффузии $\kappa_{S},-$ составляет $\delta_{\omega}^{v}=\sqrt{\mathrm{v} / \omega}$ в полях скорости, $\delta_{\omega}^{\kappa_{T}}=\sqrt{\kappa_{T} / \omega}-$ температуры и $\delta_{\omega}^{\kappa_{S}}=\sqrt{\kappa_{S} / \omega}-$ солености.

Следует отметить, что лигаменты - волокна и прослойки - наиболее распространенные и универсальные компоненты течений, которые появляются с началом действия возмущающих факторов и связывают атомно-молекулярные и макроскопические процессы. Соответственно, в режиме установления их толщина растет со временем от размеров атомно-молекулярных кластеров до масштабов наблюдаемых макроскопических компонентов, равных $\delta_{\Delta t}^{v}=\sqrt{v \cdot \Delta t}$ в полях скорости, $\delta_{\Delta t}^{\kappa_{T}}=\sqrt{\kappa_{T} \cdot \Delta t}-$ температуры и $\delta_{\Delta t}^{\kappa_{S}}=\sqrt{\kappa_{S} \cdot \Delta t}-$ солености. Расчет полей внутренних волн и лигаментов, образующихся при периодическом движении кольца по поверхности вертикального цилиндра, установленного в стратифицированном океане или атмосфере, проведен в [5].

В слабонелинейном приближении все компоненты течений начинают взаимодействовать между собой и порождать новые волны и сопутствующие лигаменты. При этом взаимодействующие пучки внутренних волн, как и взаимодействующие лигаменты, порождают все структурные компоненты - и крупные (новые ан- и субграмонические волны), и тонкие прослойки - лигаменты, и вихри [6]. Степень выраженности определенных структурных компонентов определяется геометрией и энергетикой изучаемого течения. Процессы формирования прослоек в непрерывно стратифицированной жидкости наблюдались как в областях пересечения одночастотных пучков от разных концов одного источника [7], так и разночастотных, генерируемых независимыми источниками [8] и в других работах. Учет в задаче распространения пучка внутренних волн тонкоструктурных компонентов позволяет рассчитывать амплитуды волн в жидкости с произвольным распределением плотности, в частности, при отражении от критического уровня, на котором совпадают частоты волны и локального значения периода плавучести [9]. Различия в рассчитанных и независимо измеренных позднее значениях скорости не превышают нескольких процентов [9, 10].

Собственные масштабы структурных компонентов течений, определяющие требования к программам расчета и методикам лабораторного моделирования природных процессов, были учтены при создании ком- 
плекса Уникальных исследовательских установок «ГФК ИПМех РАН» и методик моделирования широкого круга стратифицированных течений, волн, вихрей, процессов генерации, рассеяния и отражения звука [11]. Размеры области наблюдения, чувствительность и разрешающая способность оптических инструментов (включающих теневые приборы типа ИАБ-458), акустических и контактных приборов позволяют изучать широкий класс гравитационных (поверхностных и внутренних), капиллярных, звуковых волн, процессов генерации и взаимодействия между собой, а также с течениями, следами и вихрями. В серии проведенных опытов изучалось рассеяние высокочастотных звуковых пакетов с частотой заполнения 1 МГц с путными следами за буксируемыми телами или бегущими вихрями, а также процессы генерации звуковых пакетов свободно падающими в жидкость каплями.

При изучении процессов переноса вещества выяснилось, что лигаменты играют важную роль в капельных течениях, где наблюдается дискретное распределение вещества равномерно окрашенной капли в форме тонких волокон по непрерывной поверхности каверны и венца [12]. Научный и прикладной интерес представляет изучение роли тонких компонентов в капиллярных и акустических явлениях, где сопутствующие лигаменты играют важную роль в образовании и разрывах газовых полостей, сопровождающихся излучением звуковых пакетов [13].

Акустика импакта капли. Для уточнения механизмов генерации звука в данных опытах одновременно с регистрацией звуковых сигналов микрофоном и гидрофоном проводилась высокоскоростная видео- и высокоразрешающая фото регистрация картины течений.

На рис. 1 приведены типичные синхронно регистрируемые фонограммы гидрофона и микрофона, сопровождающие падение в бассейн с частично дегазированной водой одиночной капли воды диаметром $D=0.5$ см, со скоростью $U=4.0 \mathrm{~m} / \mathrm{c}$ (числа $\mathrm{Re}=U D / v$, Фруда $\mathrm{Fr}_{d}=U^{2} / g D=330 ;$ Бонда $\mathrm{Bo}=g D^{2} / \gamma=3.4 ;$ Онезорге $\mathrm{Oh}=v / \sqrt{\gamma D}=0.0016 ; \quad$ Вебера $\mathrm{We}=U^{2} D / \gamma=1100, g-$ ускорение свободного падения, $\gamma-$ коэффициент поверхностного натяжения, нормированный на плотность воды). В сигнале гидрофона (кривая 1) выражен ударный импульс I, последующий спад и подъем с возвышением венца. После продолжительной паузы в момент $t=146.8$ мс наблюдается группа гидродинамических возмущений давления, в конце которой регистрируется звуковой пакет II $(t=165$ мс). Пакет III появляется при $t=200.4$ мс. 


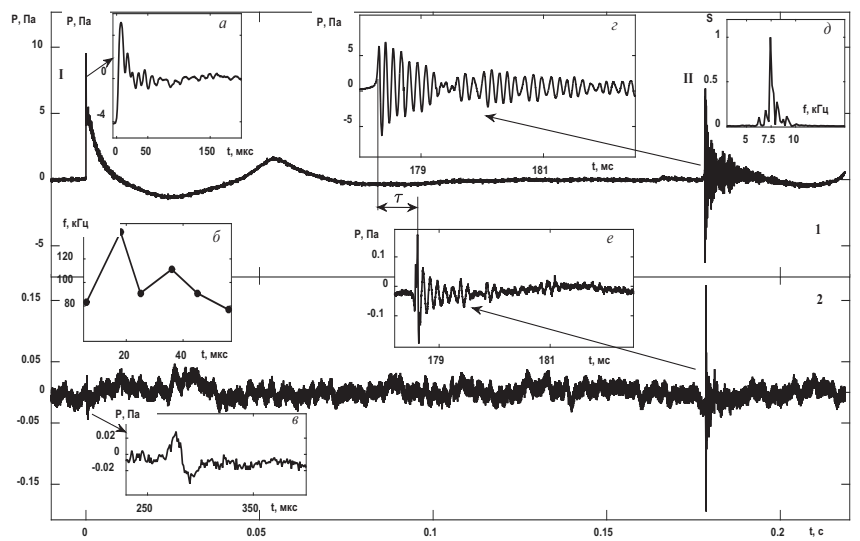

Рис. 1. Фонограммы акустических пактов импакта капли: 1 - гидрофона, 2 - микрофона.

В развертке сигнала гидрофона I на вставке а выражен начальный импульс и высокочастотные затухающие осцилляции на спадающем участке. Максимум спектра сигнала быстро сдвигается с частоты $f=$ 140 кГц к $f=80$ кГц $\tau=27$ мс (вставка б). Основной сигнал гидрофона II (развертка на вставке г) начинается с резкого нарастания, которое переходит в немонотонно спадающие осцилляции с частотой $f=7.5$ кГц (спектр на вставке д). Здесь к основному максимуму примыкают боковые лепестки на частотах $f=6.8$ и 8.2 кГц.

В сигнале микрофона (рис. 1, кривая 2) начальный импульс представлен менее контрастно, фактически одной полуволной, показанной на врезке в. Шумовая дорожка здесь более выражена, чем в сигнале с гидрофона в силу большего значения коэффициента усиления и акустического фона лаборатории. Запаздывание $\tau$ сигнала с микрофона (врезка е) соответствует времени прохождения по воздушной трассе от источника к микрофону. Спектральные максимумы сигналов с гидрофона и микрофона совпадают, что подтверждает единство источника звука в обеих средах.

При неизменных условиях характер излучения и параметры акустических пакетов значительно менялись от опыта к опыту. Одной из вероятных причин наблюдаемой нестационарности сигнала являются вариации формы капли. Эксперименты показали, что при отрыве возбуждаются и собственные колебания капли, и короткие капиллярные волны на ее поверхности, активно влияющие на взаимодействие с потоком воздуха и форму в момент контакта с принимающей жидкостью. 
Детальный анализ видеограмм показал, что генерация первичного акустического пакета происходит одновременно с касанием жидкости каплей и выбросом тонкой кольцевой пелены. На утолщенной внешней кромке пелены располагаются тонкие радиальные струйки - спайки, с вершин которых выбрасываются последовательности мелких капелек. Далее в жидкости начинает формироваться каверна с выступающим кольцевым венцом на краю. На верхней кромке венца - шевроне - располагаются заостренные зубья, к вершинам которых примыкают собственные тонкие струйки, также выбрасывающие капельки с вершин. Со временем угол наклона траекторий струек к горизонту растет, как и диаметры капелек.

Образование первичной кольцевой пелены и спаек связано с освобождением доступной потенциальной поверхностной энергии части потенциала Гиббса, обусловленной анизотропией действия атомно-молекулярных сил в областях с большими градиентами плотности и концентрации компонентов. Поверхностная энергия заключена в тонком приповерхностном слое толщиной порядка размера молекулярного кластера $\delta \sim 10^{-6}$ см. При скорости падения порядка $U \sim 10^{2}$ см/с слияние слоев под действием атомно-молекулярных процессов происходит за время $\tau_{\sigma} \sim 10^{-8}$ с. Полностью капля сливается достаточно долго - за время, превышающее $\tau_{d}=D / U \sim 10^{-3}$ с.

Освобожденная энергия, привязанная к веществу, остается «замороженной» в первичном лигаменте - тонком «двойном» слое в окрестности уничтоженных свободных поверхностей, где формируются большие возмущения температуры, давления и скорости течений. Именно на внешней кромке «двойного» слоя, выходящей из общего течения, образуются спайки. Вследствие вязкого вовлечения и формирования поперечных маленьких вихрей, толщина двойного слоя быстрого течения постепенно растет, что проявляется и в росте толщины пелены, и в размерах вылетающих брызг. Более детально анализ перестройки картины распределения потенциала Гиббса и образования лигаментов проведен в [13].

С полным погружением капли прекращается рост глубины каверны и высоты венца. Начинается фаза схлопывания каверны и расплывания венца - продолжение роста его диаметра при уменьшении высоты. При этом поверхность жидкости сглаживается. Вокруг венца образуется пакет гравитационно-капиллярных волн и семейство лигаментов, формирующих совместно с остатком двойного слоя сложную картину тонких течений. Лигаменты деформируют стенки каверны, отрывают и перемещают газовые пузырьки. 
Резкое усложнение структуры поверхности наблюдается в фазе образования всплеска, поверхность которого деформируют тонкие шипы, разделенные глубокими впадинами. Далее на вершине всплеска формируется капля с выглаженной поверхностью, опирающаяся на цилиндрический столбик, переходящий в коническое основание, окруженное группой коротких капиллярных волн. Со временем, когда капля отрывается от вершины, к основанию всплеска бежит группа капиллярных волн. Погружающийся всплеск формирует новую каверну конической формы, боковые поверхности которой покрыты короткими капиллярными волнами.

Падающая капля, которая вначале касается вершин гребней, перекрывает выход для сохраняющихся во впадинах воздушных прослоек, которые частично сливаются и образуют полости сложной формы. Газовые полости отрываются от остатка каверны и разрываются на фрагменты тонкими приповерхностными течениями. В некоторых случаях полости начинают осциллировать и генерировать основную группу резонансных звуковых пакетов с несущей частотой в диапазоне от 7 до 22 кГц.

Погружение первичной капли заканчивается формированием каверны конической формы, при схлопывании которой в воздух выбрасывается сравнительно тонкая и быстрая струйка (стример), распадающаяся на несколько капелек. Скорости капелек сравнительно быстро падают с уменьшением их номера в расположении в последовательности вылета. Образование стримера, как и его погружение, также сопровождается генераций очередной группы капиллярных волн и дополняющих лигаментов.

Новые группы кольцевых капиллярных волн образуются при падении капелек, ранее выброшенных с вершины стримера. Все группы капиллярных волн дополняются быстрыми лигаментами. Тонкие течения дополнительно ускоряются флуктуациями давления в двойных энергетически насыщенных слоях, возникающих при уничтожении свободной поверхности возвращающихся капелек. Поскольку скорость их контакта меньше, чем у первичной капли, меньше и предельные значения скорости и больше толщины возникающих течений, отрывающих газовые полости от каверн и разрывающих их на фрагменты. Именно в момент разрыва тонкого газового мостика, соединяющего отрывающийся пузырек от полости или другого пузырька, формируется звуковой пакет. Частоты излучаемого звука и первой резонансной моды возникающего сферического пузырька обычно совпадают. Длительность звучания определяется временем перестройки формы 
угловатой газовой полости в сфероидальную на последней стадии звучания. Излучаются и монотонно затухающие моночастотные, и промодулированные, и многочастотные звуковые пакеты.

Теоретический и практический интерес представляет детальное изучение свойств лигаментов - сингулярных компонентов акустических волн, учет которых позволит адекватно решать задачи распространения звука в сложных гидрологиях, отражения от твердых границ или свободной поверхности, более точно оценивать эффекты диссипации.

\section{Заключение}

Полные решения системы фундаментальных уравнений описывают волны и дополняющие семейства лигаментов, определяющих тонкую структуру течений. Продуктами нелинейных взаимодействий всех компонентов полного решения являются новые семейства лигаментов, субили ангармонических волн и вихрей.

Лигаменты образуют структуры всех видов течений - нестационарных, периодических, установившихся. В приближении однородной жидкости система уравнений вырождается и для сжимаемых, и для несжимаемых сред. Лигаменты играют важную роль в процессах формирования акустических пакетов импакта капли, генерации, распространения, отражения и затухания звуковых волн.

Методики численного и лабораторного моделирования на основе системы фундаментальных уравнений обеспечивают возможность полного сравнения расчетов и экспериментальных данных.

Работа выполнена при финансовой поддержке РФФИ (грант 18-0500870). Опыты проведены на стендах Уникальной исследовательской установки «ГФК ИПМех РАН».

\section{Литература}

1. Ландау Л.Д., Лифшиц Е.М. Гидродинамика. Теоретическая физика: Т. VI. М.: Наука, $1986.736 \mathrm{c}$.

2. Chashechkin Yu.D. Singularly perturbed components of flows - linear precursors of shock waves // Math. Model. Nat. Phenom. 2018. Vol. 13. No. 2. P. 1-29.

3. Чашечкин Ю.Д., Кистович А.В. Классификация трехмерных периодических течений в жидкости // Доклады АН. 2004. Т. 395. №. 1. С. 55-58.

4. Бардаков Р.Н., Васильев А.Ю., Чашечкин Ю.Д. Расчет и измерения конических пучков трехмерных периодических внутренних волн, возбуждаемых вертикально осциллирующим поршнем // Известия РАН. Механика жидкости и газа. 2007. № 4. С. 117-133.

5. Кистович А.В. и Чашечкин Ю.Д. Тонкая структура конического пучка периодических внутренних волн в стратифицированном океане и атмосфере // Известия РАН. Физика 
атмосферы и океана. 2014. Т. 50. №. 1. С. 117-125.

6. Кистович Ю.В., Чашечкин Ю.Д. Новый механизм нелинейной генерации внутренних волн // Доклады АН. 2002. Т. 382, № 6. С. 772-776.

7. Chashechkin Yu.D. Visualization of singular components of periodic motions in a continuously stratified fluid // J. of Visualization. 2007. Vol. 10. No. 1. P. 17-20.

8. Teoh S.G., Ivey G., Imberger J. Laboratory study of the interaction between two internal wave rays // J. Fluid Mech. 1997. Vol. 336. P. 91-122.

9. Kistovich Yu.V., Chashechkin Yu.D. Linear theory of beams internal wave propagation an arbitrarily stratified liquid // J. Appl. Mech. Tech. Phys. 1998. Vol. 39, No. 5. P. 302-309.

10. Paoletti M.S., Swinney H.L. Propagating and evanescent internal waves in a deep ocean model // J. Fluid Mechanics. 2012. Vol. 706. P. 571-583. DOI: 10.1017/jfm.2012.284.

11. УИУ «ГФК ИПМех РАН): Гидрофизический комплекс для моделирования гидродинамических процессов в окружающей среде и их воздействия на подводные технические объекты, а также распространения примесей в океане и атмосфере. http://www. ipmnet.ru/uniqequip/gfk/\#equip.

12. Чашечкин Ю.Д. Эволюция тонкоструктурного распределение вещества свободно падающей капли в смешивающихся жидкостях // Известия РАН. Физика атмосферы и океана. 2019. Т. 55. № 3. С. 285-284.

13. Чашечкин Ю.Д., Прохоров В.Е. Эволюция структуры акустических сигналов, вызванных ударом падающей капли о жидкость // Акустический журнал. 2020. Т. 66. № 4. C. $377-390$.

\title{
Yu.D. Chashechkin
}

\section{LIGAMENTS AND WAVES IN PERIODIC CURRENTS}

\author{
Institute for Problems in Mechanics. A.Yu. Ishlinsky RAS \\ Vernadsky prospect, 101/1, Moscow, Russia, 119526, \\ Tel.: 8(495)434-00-17,E-mail: ipmnet@ipmnet.ru
}

The results of theoretical and laboratory studies of the dynamics and structure of periodic flows - capillary and acoustic waves of a drop impact on the basis of a system of fundamental equations of fluid mechanics are presented. The system includes equations of state for the Gibbs potential and its derivatives - thermodynamic parameters, as well as equations for the transfer of matter, momentum, and energy. The solution is constructed by the methods of the theory of singular perturbations taking into account the compatibility condition. Complete solutions of the problems of propagation of linear acoustic, internal and hybrid waves are presented. For the first time, both regular (wave) and singular solutions characterizing ligaments - thin fibers and interlayers - are considered. The processes of formation of structures of two-dimensional and three-dimensional periodic and associated internal waves are traced. In flows generated in a liquid by freely falling drops, the connection of capillary waves with the processes of emission of sound packets is traced. 
Keywords: ligaments, waves, dynamics and structure of flows, internal waves

Acknowledgment: This work was supported by the Russian Foundation for Basic Research (grant 18-05-00870). The experiments were carried out at the stands of the Unique research facility "GFK IPMech RAS".

\section{References}

1. Landau L.D., Lifshitz E.M. Hydrodynamics. Theoretical physics, Vol. VI, Moscow: Science, $1986,736 \mathrm{p}$.

2. Chashechkin Yu.D. Singularly perturbed components of flows - linear precursors of shock waves. Math. Model. Nat. Phenom, 2018, Vol. 13, No. 2, pp. 1-29.

3. Chashechkin Y.D. and Kistovich A.V. Classification of three-dimensional periodic flows in a fluid. Dokl. AN, 2004, Vol. 395, No. 1., pp. 55-58.

4. Bardakov R.N., Vasiliev A.Yu., and Chashechkin Yu.D. Calculation and measurement of conical beams of three-dimensional periodic internal waves excited by a vertically oscillating piston. Izvestiya RAN. Mechanics of liquid and gas, 2007, No. 4, pp. 117-133.

5. Kistovich A.V. and Chashechkin Yu.D. Fine structure of a conical beam of periodic internal waves in a stratified ocean and atmosphere. Izvestiya RAN. Physics of the atmosphere and ocean, 2014, Vol. 50, No. 1, pp. 117-125.

6. Kistovich Yu.V. and Chashechkin Yu.D. A new mechanism of nonlinear generation of internal waves. Dokl. AN, 2002, Vol. 382, No. 6, pp. 772-776.

7. Chashechkin Yu.D. Visualization of singular components of periodic motions in a continuously stratified fluid. J. of Visualization, 2007, Vol. 10, No. 1, pp. 17-20.

8. Teoh S.G., Ivey G., and Imberger J. Laboratory study of the interaction between two internal wave rays. J. Fluid Mech, 1997, Vol. 336, pp. 91-92.

9. Kistovich Yu.V. and Chashechkin Yu.D. Linear theory of beams internal wave propagation an arbitrarily strati fi ed liquid. J. Appl. Mech. Tech. Phys, 1998, Vol. 39, No. 5, pp. 302-309.

10. Paoletti M.S. and Swinney H.L. Propagating and evanescent internal waves in a deep ocean model. J. Fluid Mechanics, 2012, Vol. 706, pp. 571-583, doi: 10.1017 / jfm.2012.284.

11. UIU «GFK IPMech RAS»: Hydrophysical complex for modeling hydrodynamic processes in the environment and their impact on underwater technical objects, as well as the spread of impurities in the ocean and atmosphere, http://www.ipmnet.ru/uniqequip/gfk/\#equip.

12. Chashechkin Yu.D. Evolution of the fine-structured distribution of the substance of a freely falling drop in miscible liquids. Izvestiya RAN. Physics of the atmosphere and ocean, 2019, Vol. 55, No. 3, pp. 285-284.

13. Chashechkin Yu.D. and Prokhorov V.E. Evolution of the structure of acoustic signals caused by the impact of a falling drop on a liquid. Acoustic journal, 2020, Vol. 66, No. 4, pp. 377-390. 